\title{
QCD string from D0 branes
}

\author{
Marco Billó, Michele Caselle* \\ Dipartimento di Fisica Teorica, Università di Torino \\ and Istituto Nazionale di Fisica Nucleare - sezione di Torino, \\ Via P. Giuria 1, I-10125 Torino, Italy \\ E-mail: billo, casellelto.infn.it

\section{Martin Hasenbusch} \\ Dipartimento di Fisica dell'Università di Pisa and I.N.F.N., \\ Largo Bruno Pontecorvo 3, I-56127 Pisa, Italy \\ E-mail: Martin.Hasenbusch@df.unipi.it

\section{Marco Panero} \\ School of Theoretical Physics, Dublin Institute for Advanced Studies, \\ 10 Burlington Road, Dublin 4, Ireland \\ E-mail: panero@stp.dias.ie
}

We report the results of a set of high precision simulations performed in the $3 \mathrm{~d}$ gauge Ising model. We evaluated the interquark potential and the first few energy levels and compared them with the predictions obtained with the effective Nambu-Goto string and with the free bosonic string. The data are precise enough to unambiguously distinguish between the free string predictions and those obtained using the N-G effective string. At large distances we find a remarkable agreement between Monte Carlo data and N-G predictions for the first excited energy level, while the free string picture is definitely excluded. As the interquark distance is decreased (and/or the finite temperature becomes higher) the Monte Carlo results show larger and larger deviations both from the N-G and from the free string predictions. In order to better understand this behaviour we rederived the effective Nambu-Goto theory result for the Polyakov loop correlator using a covariant quantization. We chose as boundary conditions those of an open string attached to two D0-branes at spatial distance $R$, in a target space with compact euclidean time. Obviously our treatment is fully consistent only in $d=26$. The extension to generic $d$ requires taking into account the Liouville mode of Polyakov's formulation. The analogy with the standard light cone calculation suggests that the contribution due to the Liouville field can be neglected for large $R$. At shorter scales, the Liouville mode cannot be neglected and its contribution to the interquark potential might be the source of the discrepancies with respect to the effective N-G results that we observe in our Monte Carlo simulations.

XXIIIrd International Symposium on Lattice Field Theory

25-30 July 2005

Trinity College, Dublin, Ireland

\footnotetext{
*Speaker.
} 


\section{Introduction}

The starting point of the effective string description of the interquark potential is to model the latter in terms of a string partition function. Choosing for instance to evaluate the potential by using the expectation value of a pair of Polyakov loops we have:

$$
G(R, L)=\left\langle P^{\dagger}(R) P(0)\right\rangle=\int[\mathscr{D} h] e^{-S_{\text {eff }}} \equiv Z(R, L),
$$

where $L$ is the length of the lattice in the compactified direction (i.e. the inverse temperature $L \equiv 1 / T$ ) and $S_{\text {eff }}$ denotes the effective action for the world sheet spanned by the string. In eq. (1.1), the functional integration is done over world sheet configurations which have fixed boundary conditions along the space-like direction, and periodic boundary conditions along the compactified, time-like direction (the Polyakov lines are the fixed boundary of the string world sheet).

The simplest and most natural string model is the Nambu-Goto one, which assumes the string action $S_{\text {eff }}$ to be proportional to the area spanned by the string world sheet:

$$
S_{\text {eff }}=\sigma \cdot \int d^{2} \xi \sqrt{\operatorname{det} g_{\alpha \beta}} .
$$

For the Nambu-Goto string with boundary conditions corresponding to fixed ends in the spatial directions (the static quark and anti-quark) Alvarez [1] (for $d \rightarrow \infty$ ) and Arvis [2], with a formal quantization, obtained the energy spectrum

$$
E_{n}(R)=\sigma R \sqrt{1+\frac{2 \pi}{\sigma R^{2}}\left(n-\frac{d-2}{24}\right)} .
$$

The partition function can thus be written as

$$
Z(R, L)=\sum_{n} w_{n} e^{-L E_{n}(R)},
$$

$w_{n}$ being the usual multiplicities of the bosonic string. The static potential coincides with the lowest energy level: $V(R)=E_{0}(R)$, and reproduces the well-known "Lüscher term" $[3,4]$ in the large $R$ limit. Indeed in this limit the Nambu-Goto effective action becomes the 2d CFT of $(d-2)$ massless, non-interacting bosons.

The derivation of eq.s (1.3) and (1.4) in [2] uses the re-parametrization invariance of the worldsheet to reach the conformal gauge (where the Nambu-Goto action is equivalent to the free string action) and the residual conformal invariance to fix a light-cone type gauge (which is sometimes denoted as "physical gauge"). This leaves the transverse modes as the only independent dynamical variables, which become oscillators upon quantization. This bosonic string model, of course, is truly consistent at the quantum level only if $d=26$ : as usual in light-cone type gauges, Lorentz invariance is otherwise broken. It was however noticed in [5] that the coefficient of the anomaly vanishes for $R \rightarrow \infty$, so that in this regime the model could be consistent.

In these last years, thanks to various remarkable improvements in lattice simulations, the effective string picture could be tested with a very high degree of precision and confidence [6-17] supporting the presence of a Lüscher-type correction in the interquark potential at large enough inter-quark distances (and low temperatures). Moreover, this result seems to be characterized by a 
high degree of universality, meaning that it does not depend on the particular gauge group under study (the same behaviour is observed in models as different as the $\mathbb{Z}_{2}$ gauge model in $(2+1)$ dimensions [13] and the SU(3) LGT in (3+1) dimensions [8]). However this result in itself is not very informative since it simply indicates that the quantum fluctuations of the flux tube behave as free bosonic degrees of freedom at large distance, but gives no insight on the nature of the effective string which describes the flux tube. To this end it would be important to be able to study the higher order corrections due to the effective string. A task which is very difficult to pursue as far as the interquark potential is concerned but becomes more feasible if one looks at the excited energy levels. The aim of this contribution is to address this point following two lines:

- First we shall discuss some recent numerical tests of the energy spectrum of the string performed in the $3 \mathrm{~d}$ gauge Ising model. The picture which emerges from these simulations is that the Nambu-Goto effective string prediction eq. (1.3) correctly describes the Monte Carlo data at large enough distance, while the free string model alone is incompatible with the numerical results. At the same time it is clear from the data that as the inter-quark distance decreases and/or the temperature increases (i.e. as the de-confinement transition is approached) large deviations from the N-G predictions appear (and also the universality mentioned above is partially lost) $[10,13,16,17]$.

- Second, we shall describe an alternative procedure to derive the effective Nambu-Goto theory, starting from the free bosonic string and using a covariant quantization [18]. This derivation makes more transparent the limits involved in the construction of the effective action starting from the string lagrangian and may help to understand the observed deviations at short distance.

\section{Numerical results}

In order to test the ability of the Nambu-Goto effective string to describe the interquark potential we concentrated on the $\mathbb{Z}_{2}$ pure lattice gauge theory in $d=3$, which is expected to provide a prototypical model for quark confinement. This choice is also supported by the observation that, in the region where the two models can be compared, the Ising and the SU(2) LGT's in (2+1) dimensions show very similar behaviours both from a qualitative and a quantitative point of view [12]. The nice feature of this choice is that by using duality one is able to obtain very precise estimates for the ratios of two subsequent Polyakov loop correlators: $G(R+1, L) / G(R, L)$. By integrating these ratios from $R=0$ to $R=R_{\text {max }}-1$ we may then directly obtain the correlator $G\left(R_{\max }, L\right)$ and hence the effective string partition function $Z\left(R_{\max }, L\right)$.

Our results are reported in figures 1 and 2 . The first plot shows the deviation of ratios of Polyakov loop correlators from the free string model, as a function of $L$. Looking at the figure, one can appreciate the agreement with $\mathrm{N}-\mathrm{G}$ at large distance and the deviations as $L$ decreases. Thanks to the peculiar geometry of the observable (since $L \equiv 1 / T$ as $L$ decreases we move toward the deconfinement transition where the higher order terms in the string action play a more and more important role) one can also appreciate the difference between the $\mathrm{N}-\mathrm{G}$ predictions and the free string ones. 
As anticipated above, this difference is better appreciated in fig. 2 in which we plotted the relative deviation of the first energy gap $E_{1}-E_{0}$ with respect to the free string prediction, as a function of the interquark distance. The data disagree from the $\mathrm{N}-\mathrm{G}$ expectation for low values of $R$ and then nicely converge toward it as $R$ increases. It is interesting to observe that our data perfectly agree, for low values of $R$, with those reported in [17] where in fact a disagreement with respect to the N-G picture was claimed. We confirm this disagreement at short distance, but being able to extend our analysis to larger values of $R$ we can confirm (in agreement with the above observations) that at large distances the N-G picture is fully restored. The solid, horizontal line in fig. 2 represents the free string prediction, which is clearly excluded for all values of $R$.

More details can be found in the original papers $[10,13,18]$.

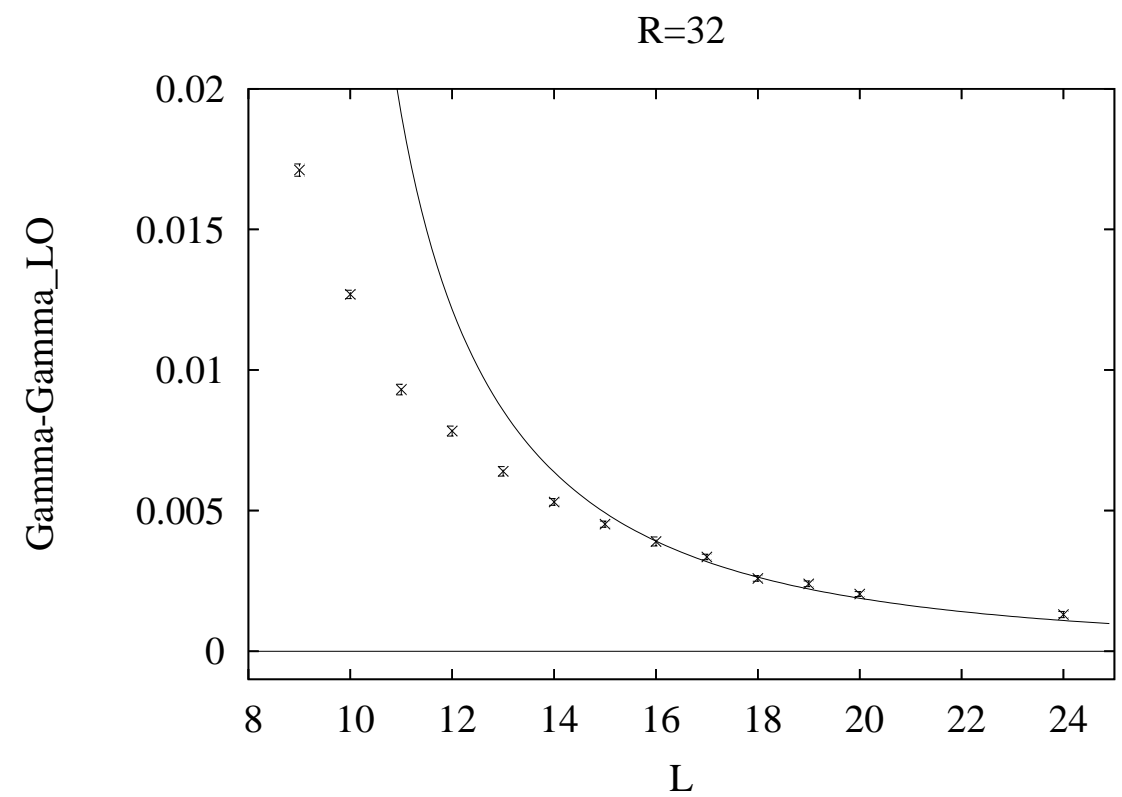

Figure 1: Monte Carlo results for the Polyakov loop correlators in the (2+1) dimensional gauge Ising model $\left(\beta_{\text {spin }}=0.226102\right)$. The data are taken at a fixed value $R=32$ of the interquark distance and a varying size $(8<L \leq 24)$ of the lattice in the time direction. The figure shows the deviation of $\Gamma$ (the ratio $G(R+1) / G(R)$ of two correlators shifted by one lattice spacing, see [13] for details) with respect to the asymptotic free string expectation $\Gamma_{L O}$ (which with this definition of observables corresponds to the straight line at zero). The curve is the Nambu-Goto prediction for this observable. Notice the remarkable agreement in the range $16 \leq L \leq 24$, which is not the result of a fitting procedure: in the comparison reported in the figure there is no free parameter (taken from fig. 3 of ref. [13]).

\section{Effective string from D0 branes}

The Nambu-Goto effective string spectrum of eq. (1.3) can also be obtained following an alternative route, using the reparametrization invariance to reach the conformal gauge (where the $\mathrm{N}-\mathrm{G}$ action is equivalent to the free string action) and then quantizing the model by using the so called covariant quantization. The boundary conditions of the string are described in modern terms as those of an open string attached to two D0-branes (which play the role of the Polyakov 


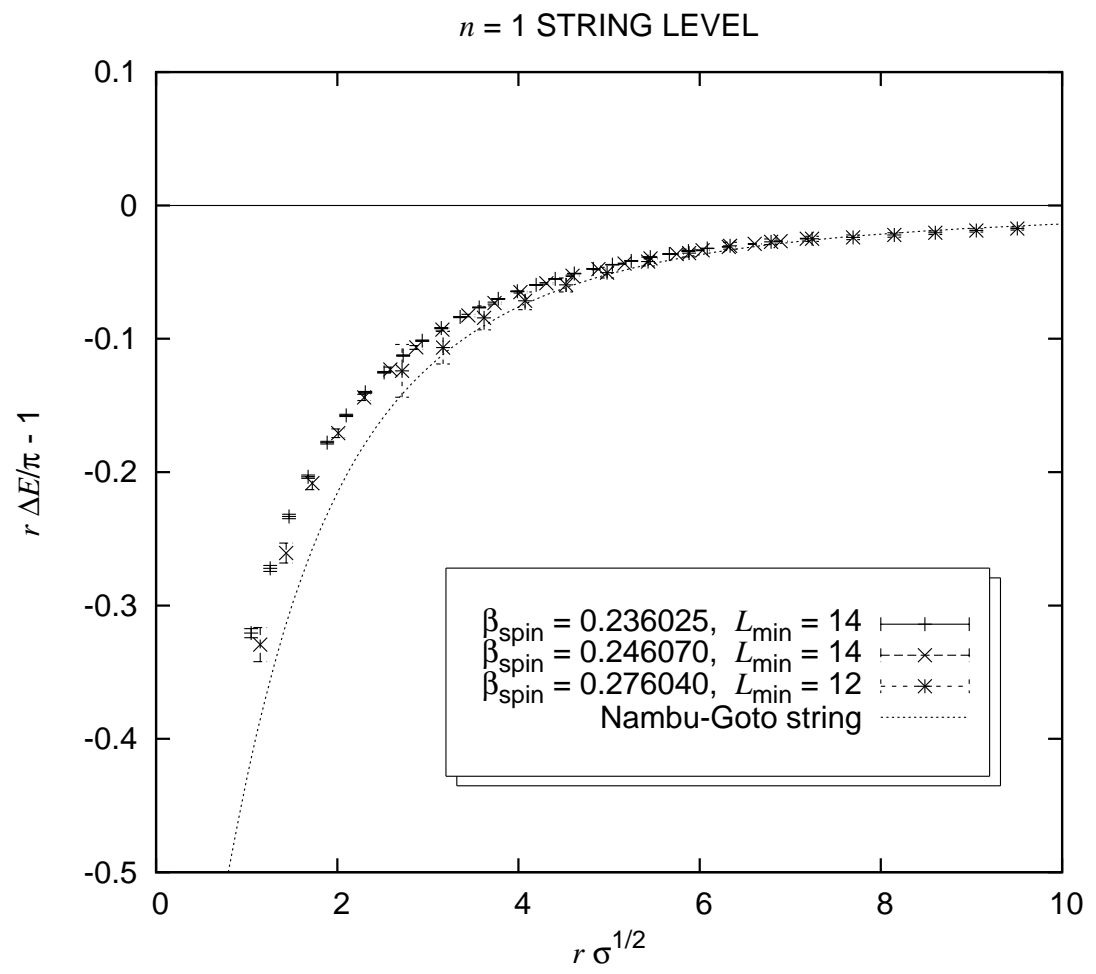

Figure 2: Relative deviation of the first energy gap $E_{1}-E_{0}$, with respect to the free string prediction, as a function of the interquark distance; results are shown for different values of $\beta_{\text {spin }}$, and are scaled in physical units.

loops) at spatial distance $R$. In this framework, the anomaly shows up with the appearance of an additional field (the so called "Liouville mode"). If one assumes that at large distance this mode can be neglected, then one can quantize the string as it is usually done for the critical bosonic string, and re-obtain all the previous results (see [18]). In particular, one finds exactly the Nambu-Goto spectrum of eq. 1.3). The major outcome of this alternative procedure is that it makes the role of the Liouville mode explicit, and it could in principle offer a clue to guess its contribution to the effective string prediction at shorter interquark distances and possibly explain the deviations observed in the lattice simulations in this regime. Besides this we think that this alternative derivation of the Nambu-Goto effective theory may have some further important advantages. Not having fixed a "physical gauge", the world-sheet duality between the open and closed channel is most evident and allows for an explicit interpretation of the free energy in terms of tree level exchange of closed string states between boundary states. Moreover our formulation is well suited in principle to study the contributions to the inter-quark potential from string interactions, which in our language would mean wrapping the Polyakov string on surfaces (bordered by the Polyakov loops) with handles. Finally, it opens the possibility of investigating the relevance in the gauge theory of the contributions to the free energy with different winding numbers (which appear naturally in our framework). 


\section{References}

[1] O. Alvarez, “The Static Potential In String Models,” Phys. Rev. D 24, 440 (1981).

[2] J. F. Arvis, “The Exact Q Anti-Q Potential In Nambu String Theory,” Phys. Lett. B 127 (1983) 106.

[3] M. Lüscher, K. Symanzik and P. Weisz, "Anomalies Of The Free Loop Wave Equation In The Wkb Approximation,” Nucl. Phys. B 173 (1980) 365.

[4] M. Lüscher, "Symmetry Breaking Aspects Of The Roughening Transition In Gauge Theories," Nucl. Phys. B 180 (1981) 317.

[5] P. Olesen, “Strings And QCD,” Phys. Lett. B 160 (1985) 144.

[6] M. Caselle, R. Fiore, F. Gliozzi, M. Hasenbusch and P. Provero, "String effects in the Wilson loop: A high precision numerical test,” Nucl. Phys. B 486 (1997) 245 [arXiv:hep-lat/9609041].

[7] M. Caselle, M. Hasenbusch and M. Panero, "String effects in the 3d gauge Ising model," JHEP 0301 (2003) 057 [arXiv:hep-lat/0211012].

[8] M. Lüscher and P. Weisz, "Quark confinement and the bosonic string,” JHEP 0207 (2002) 049 [arXiv:hep-lat/0207003].

[9] M. Lüscher and P. Weisz, "String excitation energies in SU(N) gauge theories beyond the free-string approximation,” JHEP 0407 (2004) 014 [arXiv:hep-th/0406205].

[10] M. Caselle, M. Hasenbusch and M. Panero, "Short distance behaviour of the effective string," JHEP 0405 (2004) 032 [arXiv:hep-lat/0403004].

[11] M. Panero, “A numerical study of confinement in compact QED,” JHEP 0505 (2005) 066 [arXiv:hep-lat/0503024].

[12] M. Caselle, M. Pepe and A. Rago, "Static quark potential and effective string corrections in the (2+1)-d SU(2) Yang-Mills theory,” JHEP 0410 (2004) 005 [arXiv:hep-lat/0406008].

[13] M. Caselle, M. Hasenbusch and M. Panero, "Comparing the Nambu-Goto string with LGT results," JHEP 0503 (2005) 026 [arXiv:hep-lat/0501027].

[14] M. Caselle, M. Hasenbusch and M. Panero, "On the effective string spectrum of the tridimensional $\mathbf{Z}_{2}$ gauge model," in preparation.

[15] P. Majumdar, “The string spectrum from large Wilson loops,” Nucl. Phys. B 664 (2003) 213 [arXiv:hep-lat/0211038].

[16] K. J. Juge, J. Kuti and C. Morningstar, “Fine structure of the QCD string spectrum," Phys. Rev. Lett. 90 (2003) 161601 [arXiv:hep-lat/0207004].

[17] K. J. Juge, J. Kuti and C. Morningstar, “The Casimir energy paradox of the QCD string,” Nucl. Phys. Proc. Suppl. 129 (2004) 686 [arXiv:hep-lat/0310039]; "QCD string formation and the Casimir energy," arXiv:hep-lat/0401032.

[18] M. Billó and M. Caselle, "Polyakov loop correlators from D0-brane interactions in bosonic string theory," JHEP 0507 (2005) 038 [arXiv:hep-th/0505201]. 